\title{
The Development of Conceptual Framework in Physiological Anthropology
}

\author{
Masahiko Sato \\ Nagasaki Junior College
}

\begin{abstract}
From an international viewpoint, the physiological anthropology had always developed in a mosaic-like structure until the end of the nineteen-sixties. Some of the pieces of the mosaic then started to create significant elements of the theoretical concepts of this science. Generally speaking, research in physiological anthropology consists of the process of individual biology and the process of population biology. Through using these processes, physiological anthropologists have come to realize the importance of individual thinking and the inadequacy of essentialistic concept such as the ideal man, and now infer that all populations are polytypic. Physiological anthropologists have refined the conceptual framework of their science and composed a set of keywords characterizing it. These are technological adaptability, environmental adaptability, functional potentiality, whole body coordination, and physiological polytypism. These keywords are mutually interdependent and do not form any orthogonal relations. J Physiol Anthropol Appl Human Sci 24(4): 289-295, 2005 http://www.jstage.jst.go.jp/browse/jpa

[DOI: 10.2114/jpa.24.289]
\end{abstract}

Keywords: physiological anthropology, individual thinking, quality of life, environmental stress, human physiological characteristics, technological adaptability, environmental adaptability, functional potentiality, whole body coordination, physiological polytypism

\section{Introduction}

Physiological anthropology is a steadily developing science. It aims to elucidate human physiological characteristics, in a wide sense. Seen in this context, physiological anthropology belongs to the basic natural sciences. However, the fundamental results of investigations are looked to for useful information on human characteristics from many other fields, including the applied sciences and manufacturing industries. This tendency can be understood as a reflection of social needs for knowledge about human abilities to improve the quality of life. Most human beings now live in modern technological civilization which would have been absolutely unimaginable to our ancestors. Physiological anthropology is being looked to as an important source of information on human capacities in a world which is unlike anything seen in history.

Scientists in a range of fields are engaged in conducting human research which aims to improve quality of life. Of these, the practical research carried out in physiological anthropology seems to be unique in that its approach makes use of human physiological characteristics. It can be said that the pace of development in physiological anthropology is increasing not only because of social needs but also because of recent advances in the concept and methodology of this science. From an international viewpoint, physiological anthropology in its early days was different in character in different regions, and continued to develop with remnants of these regionally characters intact, like pieces of a mosaic. It was only a few decades ago that the mosaic-like characters began to balance out. Today, these mosaic traits have come to have an effective purpose, in laying the foundations of the distinct academic originality of this science.

Having its own academic nature is a prerequisite for a distinct scientific field. Every academic field must be original in its own way. The principal conditions necessary to physiological anthropology's claim to be a distinct science can be summarized by the following five keywords: technological adaptability, environmental adaptability, functional potentiality, whole body coordination, and physiological polytypism. This short article will describe the major events in the course of theoretical development and the meaning of the keywords in common use in physiological anthropology in Japan.

\section{The Mosaic Progress of Physiological Anthropology}

Physiological anthropology is one branch of anthropology. As can be seen by the fact that Aristotle used the word "anthoropologos," there has been interest in the "science of man" since ancient times. During the 18th century, when Johann Friedrich Blumenbach took an active role in the study of human races, anthropology began to develop into an independent academic field with its own unique nature. In the 19th century, anthropological associations were established one after another in France, Britain, Germany, Japan and Russia. It is well known in Japan that Professor Karl Ernst von Baer was the first president of the Russian Society of Anthropology. 
From the early stages of the academic systematization of anthropology, it was hoped that physiological anthropology would not only use valid terminology, but would also function as an important area of the science of man, together with morphological anthropology. However, physiological anthropology made slow progress mainly due to the slow development of physiological methods. This situation continued for a considerable length of time. A methodological system capable of evaluating the physiological functions of man did not develop until just before the nineteen-fifties. Even when new methods were originated, it took a long time for the instruments used for them to spread and become prevalence. Furthermore, there were large differences in research interests between physiological anthropologists. They usually determined the subjects of their research based on their own interests. Their interests had a tendency to be affected by traditions or the academic trends of their own culture, which varied considerably among the researchers. This situation resulted in differences in research trends from country to country and region to region. For example, in Western countries research concerning human behavior was dominant and measurements of energy metabolism and body temperature regulation, including heat loss or heat production, were common research techniques, while in Japan research into muscular functions was most common, and electromyography was widely held to be the main research technique.

As in other countries, physiological anthropology in Japan has its own history. At first, electromyography was used to identify the function of skeletal muscles. This type of research became popular due to the influence of the research current in those days which focused on the effect of skeletal muscles on the shape of bones. It may be more appropriate to call research of this nature functional anatomy rather than physiological anthropology, though it was not long before electromyography was used for anthropological research into motor functions. The following studies are representative of physiological anthropology in those days: the physiological manifestation of differences in vocalization between the sexes (e.g., Tokizane and Kondo, 1954), electromyographic analysis of muscle fatigue (e.g., Sato, 1959) and of the neuro-muscular mechanism of skilled movements (e.g., Sato, 1963), neuromuscular mechanisms as seen by electromyogram (e.g., Sato, 1964). Besides electromyography, other electro-physiological methods such as electrocardiography (Sato and Tsuruma, 1967) and electroencephalography (Hayami et al., 1968) were used in physiological anthropology. Electroencephalography was not restricted to spontaneous brain wave, but was also used to research event related potentials such as the rise and fall of $\mathrm{N}_{1}-\mathrm{P}_{2}$ or the latency of $\mathrm{N}_{100}$ (Hayami et al., 1968). This type of electro-physiological research was seldom reported in this science outside Japan, though there were rare exceptions (e.g., Svorad, 1968). Physiological anthropology developed in different countries and regions with different approaches using specific methods and instruments, so from an international viewpoint, it can be said that this science continued to develop in a sort of mosaic pattern until the end of the ninety sixties.

\section{The Integration of the Mosaic of Researches}

The 8th International Congress of Anthropological and Ethnological Sciences, held in 1968, included a session on physiological anthropology for the first time. One of the most remarkable impressions from this session was the differences in research trends between countries. That is, the mosaic-like development of physiological anthropology was recognized. The difference between Western countries and Japan was particularly striking. The articles from Western countries were mostly concentrated into human adaptability to environments (e.g., Alexeeva, 1968) or human variability (e.g., Cristescu et al., 1968) and those from Japan focused on studies of neural mechanisms using electro-physiological methods (e.g., Kaizawa and Takahashi, 1968). The former gave the impression that current progress in measuring methods and instruments had not been put to effective use, and the latter failed to take populations into account. The difference between Western countries and Japan was not limited to the difference in research fields, with neural mechanisms studied in Japan and metabolic functions studied in the West, but also involved a lack of harmony in the theoretical conception of physiological anthropology.

After the 8th International Congress, a number of changes occurred in physiological anthropology. The changes were different in different countries and regions. They were drastic in some countries and was slight and difficult to recognize in others. Researchers who had been slow to adopt the latest technologies started to try to use more advanced techniques or devices, and those who had concentrated on research into neuro-muscular functions tried to widen the subjects of their research into a range of physiological functions, including environmental adaptability (e.g., Sato and Katsuura, 1973) and work capacity (e.g., Sato et al., 1974). Many physiological anthropologists appeared to have been influenced by the research activities of other researchers who presented unfamiliar and different concepts and methods. However, no international cooperation or exchanges to accelerate those movements appeared immediately after the Congress. The change from the mosaic pattern of development to the integration and systematization of physiological anthropology progressed within the different countries and regions.

Physiological anthropology in Japan underwent rapid changes. The research subjects were extended to various fields including human adaptability to environments and human variability. In particular, the influence on human physiological characteristics of the mechanized world of modern technological civilization became one of the most popular research subjects. The expansion of research subjects brought about an increase in the number of researchers in this science. The membership of the Japan Society of Physiological Anthropology gradually increased and reached about one 
thousand. This is larger than the membership of the Anthropological Society of Nippon (Japan Society of Physical Anthropology), which was founded about one hundred years before the Japan Society of Physiological Anthropology. Among the factors which contributed the growth of the Japan Society of Physiological Anthropology, the most noteworthy was the academic characteristics of physiological anthropology, that is, the conceptual framework of this science which was beginning to be formed from the parts of the mosaic which had developed separately in the different countries and regions. The concepts were formed by the pieces of the mosaic melting together.

\section{Physiological Attributes and Individual Thinking}

Humans are equipped with the ability to feel and know over an unimaginably wide range. For instance, humans have sensibility (kansei) as well as intellect. The sensibility is the ability to recognize by intuition, while intellect is the ability to recognize with symbols, including language. Recognition through sensibility does not depend upon language and exhibits a sort of multi-faceted character. It makes it possible to feel and know something immediately without any intervening stage or the conscious use of reasoning. It is sometimes used to get an all-encompassing wide view and sometimes to get a sharp, focused idea. These views and ideas influence the various systems of physiological functions. Recognition through intellect, in contrast, uses symbols such as language to organize information. This organizing system of information is an indispensable part of thinking and understanding. It is really the source of intellect. However, recognition using this organizing system of information tends to restrict one's view of reality. Physiological anthropologists have found that the results of research obtained with language responses are different from those obtained with physiological responses. This difference has been found in numerous cases and it has been concluded that it comes from the fact that physiological responses included not only responses from the intellect but also those from sensibility, and reflect a reality that exists beyond linguistic expressions. Through this experience, physiological anthropologists have come to give a much higher priority to physiological measurements than to linguistic responses.

In general, research in physiological anthropology begins with physiological measurements of individuals. This procedure is literally a study of individual biology. After this, the physiological data are treated statistically, and the population is analyzed biologically. It is not difficult to measure and analyze physiological responses, owing to recent advances in measurement techniques and instruments. The most advanced equipment and methods are used in almost all areas of physiological anthropology. The difficulties are believed to lie in "the individual thinking".

It is a general tendency that as the accuracy of measurements increases, the differences in physiological responses between individuals become much clearer. Faced with this fact, physiological anthropologists have become strongly convinced that there are no physiological attributes without individual differences, and that the mean value or average is only a statistical abstraction and the variation is the reality for human beings. Physiological anthropologists convince that every individual is a real and unique existence so the concept such as the ideal man lacks scientific validity. They believe that the most important methodology in physiological anthropology is closely related to individual thinking and that any kind of approach based on essentialism is quite inadequate to the elucidation of biological attributes of human beings. This thinking in physiological anthropology has contributed to the refinement of its theoretical concepts and created the keywords characterizing it, which were given in the introduction.

\section{Technological Adaptability}

Biological anthropologists traditionally have specific research fields such as the Micronesian islands, Altiplano in Peru, or the swamp areas of Hokkaido. A specific population of which individuals are similar in genetic backgrounds or in living styles may be an ideal subject for the study of interactions between population, climate and natural features, and cultural basis. However, most physiological anthropologists study people living in cities, in which the interaction between population, climate, and culture is extremely complex. They seem to decide upon the subject of their research not from the viewpoint of the academic tradition of anthropology but from the viewpoint of contributions to society, embarking on a passionate search for human characteristics in modern technological civilization. They seem to want to ask what they can do for human beings and to establish an ideal methodology which enables them to find out about not only distant ancestors or people living in a primitive state, but also people who are following a life similar to themselves.

Today, the majority of human beings live in modern cities. They walk along treeless avenues, look up at starless skies, work in dull rooms with air conditioning and artificial illumination, and are accustomed to continuous noise and vibration. Nevertheless, it seems that the seizure of nature by modern technology does not necessarily create an environment inappropriate to human life. The living environment of a modern city, which would have seemed utterly abnormal to our ancestors, has now become a normal, everyday environment. However, the human constitution is without doubt a product of the process of evolution. Man lived among the savagery of nature for about five million years after he had become man. All man's physiological functions had been created solely in order to adapt to nature in those days. Artificial life in a mechanized world has not been experienced before, and this artificial life will doubtless exert numerous irreparable influences on man's constitution. Faced with one of their most 
serious problems, physiological anthropologists want to play a significant role in adjusting these artificial living conditions to man's constitution for the welfare of mankind. Anthropology that takes pride in "the science of man" has the obligation to send the necessary information to other fields.

Modern civilizations seem to be focused on achieving comfort, avoiding exposure to stresses, and removing extreme inconveniences or difficulties from living environments. Certainly, it is quite rare in daily lives to encounter an extreme level of stress that threatens life. Acute, exotic or dramatic situations such as extraordinary high or low air temperatures no longer exist. However, less drastic, less acute, less obvious and longer lasting stresses in daily lives have a large possibility of producing unprecedented and negative influences on human constitution. The target of physiological anthropologists' studies has become these unprecedented stresses in daily life.

Modern technology has reached the level that if the necessary conditions for human beings were discovered, any condition could be achieved, in almost all cases. What is needed is information on human beings. Physiological anthropologists are conducting researching into various types of daily stresses and sending information about this to other fields. Actual examples of this are research on human adaptability to living environments, the criteria for human comfort, optimal living conditions, the effects of living patterns on physical capabilities, the physical capabilities of the elderly and living support tools, man-machine interfaces, human sensibility and fashion (e.g., Sato et al., 1999).

If most physiological anthropologists were asked what their specific research field was, they would answer that it is the city in which man goes about his modern technological life. The purpose of research is to find out the capacity of humans for going about daily life in a mechanized world made by modern technology. "Technological adaptability," an area about which information is needed desperately in almost all areas of modern society, is listed as one of the keywords which explains the academic characteristics of physiological anthropology. The theoretical stance of this science and the approach takes towards finding out about technological adaptability seems to have been admitted and accepted by the present society, judging from the increase in the amount of joint research with industry. This approach also seems to be responsible for the increase in the membership of the academic society for this science.

\section{Environmental Adaptability}

Since old times, human adaptability to the environment has been one of the main subjects of physiological anthropology. As shown in the investigations of IBP projects, the main concerns of the research were the ability of humans to tolerate acute, exotic or dramatic environmental stresses such as air temperatures higher than body temperature, the low air temperatures encountered in arctic areas, or the hypoxic conditions experienced in Altiplano or the Himalayas. Today, physiological anthropologists investigate human adaptability not only to extreme environmental stresses but also to the less extreme stresses present in daily life. For example, subjects investigated besides the physiological effects of stress due to extremely high or low air temperature (e.g., Sato et al., 1983), or hypoxic stress (e.g., Sato and Sakate, 1974), have included the physiological influences of dairy stress (e.g., Sato et al., 2000), including the non-visual effect of the color temperature of illuminations (e.g., Sato and Noguchi, 1993) have been investigated. This enlargement of the range of research topics brought about theoretical developments in physiological anthropology. Less obvious and long lasting types of stresses in modern society have been seldom watched before. It is commonly understood that natural selection cannot possibly continue to adapt to an environment that no longer exists, any more than it can adapt the human population to environments that have not yet been created. Physiological anthropologists have begun to be really concerned about the possibility of the loss of inherent qualities from the mind and body in an environment that is so mild and soothing that it makes life effortless. What is more, the modern technology that allows man to change his environment with the intention of minimizing effort is capable of bringing about an unimaginably dangerous environment. For this reason, physiological anthropologists consider that adaptability to unpredictable dangers is a basic requisite among the intrinsic conditions for life. They have been therefore concerned with the possibility that the ability to completely adapt to the present mechanized environment may conflict with success in environments in the future, and have pointed out the risk of adapting to the present environment created by modern technological civilization alone. They warn that this state of adaptation creates a false sense of sufficiency which has no value outside the confines of a given era. Physiological anthropologists affirm that the state of adaptation must be replaced by the adaptability. The term "environmental adaptability" is listed as one of the keywords which characterize physiological anthropology for the reasons given above.

Adaptability consists of many phenotypic processes whose genetic basis is provided by balanced polymorphism. Physiological anthropologists are investigating adaptability by performing physiological analysis of potential capacities to withstand a range of environmental influences. They expect this approach to yield quantitative information on potential capacities.

\section{Functional Potentiality}

Various kinds of tolerances have been investigated in physiological anthropology. The areas investigated have included heat tolerance, hypoxic tolerance, tolerance to air pollution, tolerance to hard work, tolerance to malnutrition, tolerance to noise and vibration, tolerance to emotional disturbances, and tolerance to mental stress. 
It has been recognized that tolerances always have reserves of potential. For example, the tolerance to heat can easily be changed by activating potential reserves. It has been confirmed that acclimatization to heat over only two weeks produced a remarkable rise in tolerance to heat, and that after acclimatization, levels of heat tolerance are almost the same in Japanese people, Caucasians and Africans (Sato et al., 1989). Expressions of mental capacity also show similar increases with the application of habituation, learning, concentration or effort. It has been proved that one's tolerance of mental loads can be changed by the exertion of spare mental capacity (e.g., Sato, 1971). Likewise, almost all the relevant research in physiological anthropology has indicated that no adaptability can be separated from the potentiality, and thus that evaluating adaptability without considering potentiality can only lead to a false and fictitious conclusion.

The concept of adaptation brings to mind the image of passive acceptance of undesirable conditions. The adaptability which is really worthwhile to human characteristics is quite different from this. It is desirable to maintain the adaptability to both the ancient and future environments. Physiological anthropologists predict that investigations into tolerance could be an effective way to understand the potential capacities, and even imagine finding a scientific means of obtaining actual beneficial levels of adaptability to future environments.

Physiological anthropologists consider that human capacity still contains large unexplored potentialities, in both phenotypic and genotypic ways. This is why the term "functional potentiality" is listed among the keywords characterizing physiological anthropology.

\section{Whole Body Coordination}

One of the main concepts of physiological anthropology is individual thinking, as has been touched on already. As is well known, various sorts of coordinative mechanisms act within the bodies of individuals. The reciprocal innervation between the extensors and flexors during the movement of joints, and between the sympathetic and parasympathetic autonomic nervous systems, might be representative examples of this coordination. The first concern of physiological anthropologists is how the coordination is integrated within the individuals.

Coordination also acts within tissues or organs such as muscle fibers or synergists during voluntary muscular contraction. For examples, in the case of the elbow flexion, coordination occurs between $M$. biceps brachii and $M$. brachioradialis, and if the flexion is intense, the role of agonist will change between them, due to an ischemic effect from the contraction via afferent information from the proprioceptors within both the muscles. This phenomenon has been pointed out as a sort of protecting mechanism for muscle fatigue from the frequency analysis of electromyogram (Sato, 1965). Similarly, intense and long lasting mental concentration produces various sorts of activation shift among the central nervous system which gradually falls into repetitions of the spontaneous pause state. This phenomenon is thought to be produced by the coordination of many cerebral modules, though the details of the mechanisms are unknown (Sato, 1971). Coordination also occurs between different organs and systems, for example between the nervous and endocrine systems and between the respiratory and excretory systems. For example, acute mountain sickness is generally caused by a failure in the acid-base balance due to over-ventilation in a hypoxic condition, which appears as nervous symptoms. These nervous symptoms are alleviated immediately after recovery from respiratory alkalosis by an obvious increase in the volume of bicarbonate excreted owing to the accelerated functioning of the kidney (Sato, 1981).

Every sort of physiological function includes the coordination, though this is only natural. The study of the biology of individuals could not exist without considering whole body coordination. One of the difficulties in physiological anthropology lies in the system of individual thinking, with research data on minute parts of the body such as cells, organs, tissues, or systems. The difficulty often comes from a lack of knowledge to link the analysis of minute parts to the synthesis of the whole body. In particular, the mechanisms of the brain related to much of the research in physiological anthropology are mostly unknown. Research in this area is carried out as a combined effort, investigating the coordination not only between each of the modules in the brain but also between each of the systems in the whole body, and checking the state of current knowledge on brain physiology.

Physiological anthropologists also infer that the possibility for individual uniqueness hidden in physiological mechanisms is highly relevant to coordination. Investigations into coordination could clarify the nature of a number of individual differences present in biological phenomena and show the variation not to be an error to be real. The term "whole body coordination" is listed as a keyword, as it characterizes physiological anthropology from the point of view of the importance of individual thinking.

\section{Physiological Polytypism}

It is an attractive approach for physiological anthropologists to clarify the characteristics of a given population. The publication of "The Encyclopedia of the Japanese People (Sato, 2003)" was carried out not only in response to social need but also as a result of academic interest in physiological anthropology. While some very difficult work was involved in this publication, it was absolutely impossible to identify any homogeneous characteristics in the Japanese population. For example, the reports for the estimated value of the lower critical temperature, which is a popular index for cold tolerance, differed widely between researchers (Sato, 1984). The confidence ranges for mean estimated critical values were also found to be remarkably wide using the polynomial equation method (Sato et al., 1979). These results imply the 
possibility that the Japanese people consist of several different types with different cold tolerances, so that the groups studied by each research group included these different types in different ratios. In fact, the existence of different types of people whose resistance to cold varies was supposed from the increase in the ratios of heat production when mean body temperature was lowered (Sasaki, 1982). There are also different types of Japanese people who have different respiratory responses to the carbon dioxide concentration in inspired gas (Sato, 1981). The existence of different types of reaction to olfactory sensations and taste sensations has been confirmed from the differences in brain blood flow (Tsunetsugu et al., 2003), and this difference is even thought to have some relationship with personality (Tsunetsugu et al., 2004).

Faced with these various types of individual differences, physiological anthropologists have realized the uniqueness of each individual within a population. Individuals who are measured with some physiological procedure and show a value which is far from the average have completely normal physiological functions, which are the same as those who show an average value. Physiological anthropologists tend to see these measurements not as a standard value and a deviant value, but as two norms.

Human beings adapt to various environments, producing geographical and cultural variations between each other. Genetic mechanisms are at work in these processes, and every population has multiple normal genotypes. Various sorts of phenotypic process combine to create the variation. There are no groups which are not polytypic. Physiological anthropologists consider every population to have multiple normal physiological types created by phenotypic processes based on balanced polymorphism, so that elucidating the mechanism of this physiological polytypism will bring about definite progress in this science. The term "physiological polytypism" is listed among the keywords characterizing the concept of physiological anthropology.

Some of the components of the mosaic which have been observed in the developmental process of physiological anthropology have created significant elements of the theoretical concepts behind this science. This framework continues to develop as this science progresses. The concepts may be modified if some of the elements become more or less important, but at present the conceptual framework can be expressed with the five keywords given. These keywords interact in an elaborate way and none of them are orthogonal to each other, as explained above.

\section{References}

Alexeeva TI (1968) Morpho-functional population studies in some biogeochemical USSR provinces as viewed in the light of the adaptation problem. Cong Anthropol Ethnol Sci: 72-75

Cristescu M, Radu D, Lazar A (1968) Aspects correlatifs morph-fonctionels et biochimiques de la crooissance et du development des enfants et des adolescents. Cong Anthropol Ethnol Sci: 66-68

Hayami A, Ishii M, Sato H, Sato M (1968) Activation level and cortical evoked responses. Proc 8th Int Cong Anthropol Ethnol Sci: 76-77

Kaizawa J, Takahashi I (1968) The attitude of H-reflex to the repetitive stimulation and its meaning in human posture. Cong Anthropol Ethnol Sci: 77-78

Sasaki T (1982) Health and Climate. Asakura-Shoten, Tokyo, 1-197 [In Japanese]

Sato M (1959) Fatigue and exercise effects in the rapid repeating movement. J Jap EMG Soc: 110-111

Sato M (1963) An electromyographic study on skilled movements. J Faculty of Science Univ Tokyo VII (4): 323-369

Sato M (1964) The frequency components of the electromyogram led with bipolar electrodes. J Anthropol Soc Nippon 72: 92-106

Sato M (1965) Some problems in the quantitative evaluation of muscle fatigue by frequency analysis of EMG. J Anthropol Soc Nippon 73: 20-27

Sato M (1971) Textbook of Ergonomics. Koseikan, Tokyo, 324-341 [In Japanese]

Sato M (1981) The hypoxic and hyper-oxic Environments. In Kikuchi M, Sakamoto T, Sato M, Tanaka M, Yoshida K eds. The Introduction of Physiological Anthropology. Nankoudou, Tokyo, 177-225 [In Japanese]

Sato M (1984) Human Adaptation to the Climate. In Japan Society of Physical Anthropology ed. The Anthropology-its developments in multiple areas. 158-169 [In Japanese]

Sato M (2003) Encyclopaedia of the Japanese. AsakuraShoten, Tokyo, 1-718 [In Japanese]

Sato M, Jürgens HW, Narančić NS, Rudan P (2000) Current topics in physiological anthropology. Croatian Anthropol Soc, 1-129

Sato M, Katsuura T (1973) Physiological strain during high atmospheres pressure. Ergonomics 16: 315-316

Sato M, Katsuura T, Yasukouchi A (1979) The lower and upper critical temperature in male Japanese. J Human Ergol 8: $145-153$

Sato M, Matsuda K, Koujima T (1989) The adaptability to heat of young adult Japanese. Ann Physiol Anthropol 8: 25-27

Sato M, Noguchi H (1993) The effect of color temperature of lighting sources. Proc Int Symposium on Design of Amenity: 92-93

Sato M, Sakate T (1974) Combined influences on cardiopulmonary functions of simulated high altitude and graded work loads. J Human Ergol 3: 143-148

Sato M, Sakate T, Ishii M, Koga S, Katsuura T (1974) A comparison of work efficiency between urban and suburban children. J Human Ergol 3: 143-148

Sato M, Tokura H, Watanuki S (1999) Recent advances in physiological anthropology. Kyushu Univ Press, 1-401

Sato M, Tsuruma S (1967) Cardiac and muscular factors in the 
micro-vibration on the human body surface. J Anthropol Soc Nippon 75: 19-31

Sato M, Yasutaka S, Inoue K, Fukuba Y, Fujiie K, Yoshioka H (1983) The effect of air temperature on maximal oxygen intake. J Anthropol Soc Nippon 91: 377-388

Svorad D (1968) Phylogenetic precursors of human sleep: an experimental analysis. Cong Anthropol Ethnol Sci 75-76

Tokizane T, Kondo S (1954) Male voice and female voice. Human Science 6: 18-27

Tsunetsugu Y, Miyazaki Y, Morikawa T, Kamiwaki T (2003) Personality and physiological response: relationship between type A behavior pattern, trait anxiety, gender role perception and variation in cerebral blood flow change-taking chocolate stimulus as an example-. Jpn J Physiol Anthropol 8: 259-260 [In Japanese]

Tsunetsugu Y, Miyazaki Y, Morikawa T, Kamiwaki T (2004) Relationship between type A behavior pattern, trait anxiety, and variation in cerebral blood flow change. 7th Int Cong Physiol Anthropol Abstracts: 35-36

Received: January 31, 2005

Accepted: March 15, 2005

Correspondence to: Masahiko Sato, 1-44-14-705 Komaba, Meguro-ku, Tokyo 153-0041, Japan 